\title{
Route of Administration Not Applicable
}

National Cancer Institute

\section{Source}

National Cancer Institute. Route of Administration Not Applicable. NCI Thesaurus. Code C48623.

The route of drug administration is not applicable. 